University of Wollongong

Research Online

Faculty of Engineering and Information

Faculty of Engineering and Information

Sciences - Papers: Part A

Sciences

$1-1-2014$

\title{
Capability chart: A new tool for grid-tied microgrid operation
}

Athmi Jayawardena

University of Wollongong, avj998@uowmail.edu.au

Lasantha Meegahapola

University of Wollongong, lasantha.meegahapola@rmit.edu.au

Daniel Robinson

University of Wollongong, dr964@uowmail.edu.au

Sarath Perera

University of Wollongong, sarath@uow.edu.au

Follow this and additional works at: https://ro.uow.edu.au/eispapers

Part of the Engineering Commons, and the Science and Technology Studies Commons

Research Online is the open access institutional repository for the University of Wollongong. For further information contact the UOW Library: research-pubs@uow.edu.au 


\title{
Capability chart: A new tool for grid-tied microgrid operation
}

\begin{abstract}
Increasing penetration of distributed resources have enabled the development of the microgrid concept. Similar to traditional generators, microgrids can participate in the energy market to achieve technical, economical and environmental benefits. In order to facilitate the participation in energy markets, microgrid operators need to be aware of the full capability of the microgrids. This paper presents a systematic approach for developing a capability chart for a grid-tied microgrid which represents the active and reactive power capability at the grid supply point. Capability charts have been developed for two different microgrids and the impacts of different modelling aspects have been analysed under several scenarios. Furthermore, operating points of the capability charts have been verified using time-domain simulations.
\end{abstract}

\section{Keywords}

tied, grid, microgrid, tool, capability, chart, operation

Disciplines

Engineering | Science and Technology Studies

\section{Publication Details}

A. V. Jayawardena, L. G. Meegahapola, D. A. Robinson \& S. Perera, "Capability chart: A new tool for gridtied microgrid operation," in T\&D Conference and Exposition, 2014 IEEE PES, 2014, pp. 1-5. 


\title{
Capability Chart: A New Tool for Grid-tied Microgrid Operation
}

\author{
A. V. Jayawardena, Student Member, IEEE, L. G. Meegahapola Member, IEEE, \\ D. A. Robinson, and S. Perera, Senior Member, IEEE \\ School of Electrical, Computer and Telecommunications Engineering, University of Wollongong, \\ Australia.
}

\begin{abstract}
Increasing penetration of distributed resources have enabled the development of the microgrid concept. Similar to traditional generators, microgrids can participate in the energy market to achieve technical, economical and environmental benefits. In order to facilitate the participation in energy markets, microgrid operators need to be aware of the full capability of the microgrids. This paper presents a systematic approach for developing a capability chart for a grid-tied microgrid which represents the active and reactive power capability at the grid supply point. Capability charts have been developed for two different microgrids and the impacts of different modelling aspects have been analysed under several scenarios. Furthermore, operating points of the capability charts have been verified using time-domain simulations.
\end{abstract}

Index Terms-Capability chart, distribution system, grid-tied microgrid, optimisation.

\section{LIST OF SYMBOLS}

$\delta_{i} \quad$ Phase of the voltage at node $i$

$\phi_{i k} \quad$ Phase of the line admittance between node $i$ and node $k$

$\sum P_{\text {loss }}$ Total active power losses in the microgrid

$I_{i k} \quad$ Magnitude of the current between node $i$ and node $k$

$P_{g i} \quad$ Active power output from the generator at node $i$

$P_{G S P} \quad$ Active power through the grid supply point

$P_{i} \quad$ Active power through node $i$

$P_{l i} \quad$ Active power demand of the load at node $i$

$Q_{g i} \quad$ Reactive power output of the generator at node $i$

$Q_{i} \quad$ Reactive power through node $i$

$Q_{l i} \quad$ Reactive power demand of the load at node $i$

$S_{t r} \quad$ Rated apparent power of the transformer

$V_{i} \quad$ Magnitude of the voltage at node $i$

$Y_{i k} \quad$ Magnitude of the line admittance between node $i$ and node $k$

\section{INTRODUCTION}

Microgrid paradigm has gained much interest in the power industry due to the increased penetration of distributed generation ( $\mathrm{PV}$, microturbines, wind generators etc.), and storage devices (battery storage, flywheels etc.) at distribution level. Microgrids' ability to provide energy requirements to the end users during main grid outages and the capability of parallel operation with the utility to provide ancillary services improve the reliability, efficiency and reduce overall costs. Apart from the technical and economical benefits, microgrids have paved the way to achieve environmental benefits by creating zero-net-energy communities and reducing overall carbon footprint [1]. From the network perspective, grid-tied microgrids can be operated as single generating entities or as loads. Integrating microgrids into the network and building a multi-microgrid environment will allow microgrids to provide services to the upstream grid during normal grid-tied operation and emergency services to adjacent microgrids in a technically and economically feasible way. In the future, these microgrids can participate in the wholesale markets to supply energy and other ancillary services similar to conventional generators in traditional power systems [2].

Few research activities have been carried out on developing microgrid central control systems. A multi-agent control system has been proposed in [3] with the aim of optimising internal operation of a microgrid rather than optimal participation of the microgrid itself in the market. A microgrid central controller(MCC) performs the optimal resource allocation within the microgrid, however it is assumed that there is no limit to the power that can be sold or bought from the main grid. In [4], participation of microgrids in the open market has been considered enabling microgrids to buy and sell power to the main grid. In order to maximise the profit, optimisation process is carried out on every pre-defined time interval. However, in [3] and [4], the distribution network service provider (DNSP) or the distribution management system do not utilise information on the full active and reactive power capability of the microgrid for the considered time period. Previous research has focused on optimising the power availability and minimising operational costs within the distribution network while minimising active and reactive power flow at the grid supply point (GSP) [5]-[8]. However, enabling the participation in power market and ancillary services market while fulfilling the local energy demand will provide extra revenue for grid-tied microgrids. In order to facilitate this concept, MCCs or DNSPs must not only find the optimal operating solution but they must also be aware of the full capability of the microgrid at the GSP in order to make decisions in the market.

The concept of capability chart is traditionally related to synchronous machines, and represents the area of permissible operation in terms of active and reactive power availability at the machine terminal [9]. Recent research activities have been carried out on developing capability charts for wind turbines based on doubly fed induction generators (DFIG) [10], [11], grid connected PV units [12], generator-transformer units [13], and HVDC links [14]. These capability charts are considered as essential system planning tools and are widely being used in power system operation. Two different methods of deriving 
capability charts can be found in [15] and [16].

This paper presents a new approach on developing a chart for the active and reactive power capability of a microgrid at the GSP, while taking into account the generation limits, local load demands, network constraints and other technical constraints. The method for obtaining the capability chart is based on an optimisation model. Capability charts are derived using MATLAB for two microgrid models under different network configurations and validated using DIgSILENT PowerFactory. Structure of this paper is as follows; The optimisation model is presented in Section II. Section III presents the derivation of the capability chart under different scenarios for a five-bus microgrid model. A case study on a grid-tied microgrid model based on a modified version of the IEEE 13-Bus system is presented in Section IV. Discussion and conclusions are given in Section V and Section VI respectively.

\section{OPTIMISATION MODEL}

Optimisation technique is used to derive the capability chart for a grid-tied microgrid in the complex power plane. Objective function is developed to maximise the active power for a particular reactive power flow through the GSP while minimising the microgrid total active power losses. Selection of this objective function will allow the microgrid to increase revenue by power exporting while reducing the costs for network losses. Reactive power through the GSP is increased iteratively and the optimisation model is solved at each step yielding the maximum active power through the GSP. This procedure is carried out for both power import and export modes. In order to obtain the capability limits, the objective function is subjected to typical AC power flow constraints during all time periods and all network configurations.

Objective function;

$$
\operatorname{Max}\left(P_{G S P}-\sum P_{\text {loss }}\right)
$$

where, $P_{G S P}=f(V, \delta, Y)$.

Subjected to load flow equalities

$$
\begin{gathered}
P_{g i}-P_{l i}-P_{i}(V, \delta)=0 \\
Q_{g i}-Q_{l i}-Q_{i}(V, \delta)=0
\end{gathered}
$$

Inequalities will account for the limits on magnitude and phase angle of nodal voltages,

$$
\begin{gathered}
V_{i}^{\min } \leq V_{i} \leq V_{i}^{\max } \\
-\pi \leq \delta_{i} \leq \pi
\end{gathered}
$$

Distribution line capacity limits,

$$
I_{i k}(V, \delta) \leq I^{\max }
$$

Power flow between the microgrid and the main grid is limited by the coupling transformer at the GSP. Transformer power limit is incorporated as follows,

$$
\sqrt{P_{t r}^{2}+Q_{t r}^{2}} \leq S_{t r}
$$

Maximum and minimum generator power capability limits,

$$
\begin{aligned}
& Q_{g i}^{\min } \leq Q_{g i} \leq Q_{g i}^{\max } \\
& P_{g i}^{\min } \leq P_{g i} \leq P_{g i}^{\max }
\end{aligned}
$$

where

$$
\begin{aligned}
& P_{i}(V, \delta)=V_{i} \sum V_{k} Y_{i k} \cos \left(\delta_{i}-\delta_{k}-\phi_{i k}\right) \\
& Q_{i}(V, \delta)=V_{i} \sum V_{k} Y_{i k} \sin \left(\delta_{i}-\delta_{k}-\phi_{i k}\right)
\end{aligned}
$$

$$
I_{i k}(V, \delta)=\frac{\sqrt{V_{i}^{2}+V_{k}^{2}-2 V_{i} V_{k} \cos \left(\delta_{i}-\delta_{k}\right)}}{Z_{i k}}
$$

$Z_{i k}$ is the magnitude of the system impedance between node $i$ and $k$. Voltage at the GSP is maintained at $1 \angle 0^{\circ}$ pu. As the non-linear optimisation problem cannot be solved analytically, numerical solutions are obtained by using the interior-point algorithm [17] available in the optimisation solver in MATLAB.

\section{CAPABILITy CHART}

\section{A. Microgrid model-1}

Fig. 1 illustrates the microgrid model-1, comprising a 5 MVA synchronous generator (SG) based hydro power plant and a 1.5 MVA doubly fed induction generator (DFIG) based wind generator unit. Daily generation profiles of the SG and the DFIG are presented in Fig. 2-(a). The microgrid system is connected to the external grid through a $10 \mathrm{MVA}$, $69 \mathrm{kV} / 13.8 \mathrm{kV}$ transformer. Load-1 and load-2 are considered as an industrial customer (0.85 p.f.) and a commercial customer (0.85 p.f.) respectively, having load patterns on a typical weekday during the summer as illustrated in Fig. 2-(b) [18]. During this study, it is assumed that tap position in the coupling transformer is fixed. Several scenarios are undertaken and are presented in the subsections as follows; (1) the effects of capability curves of different distributed generators (DGs), (2) modelling voltage dependency of loads, (3) variation with different loading conditions, and (4) bus voltage regulation on the overall microgrid capability chart.

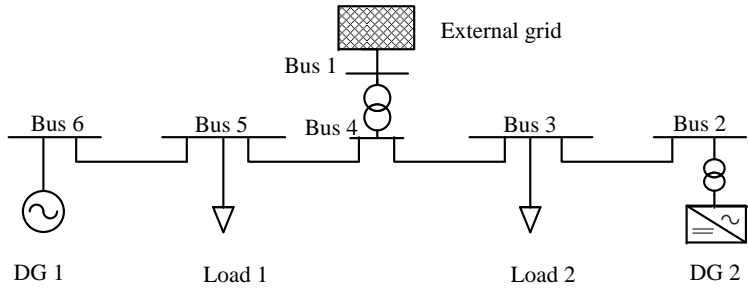

Fig. 1. Single line diagram of the microgrid model-1 


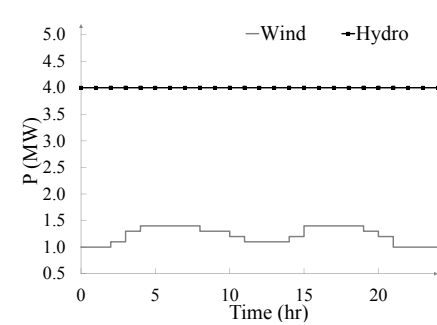

(a)

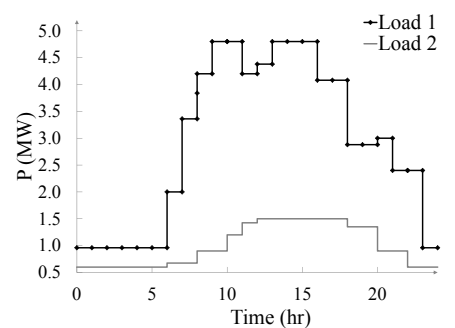

(b)
Fig. 2. (a) Daily generation profiles of the SG and DFIG, and (b) Daily load profiles for load 1 and load 2 for a typical weekday in summer

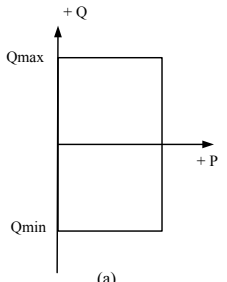

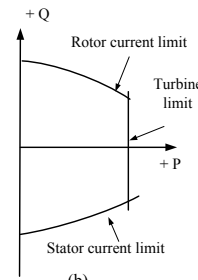

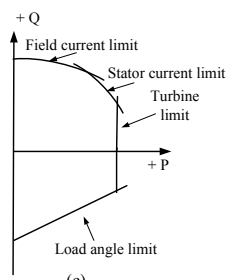

(c)
Fig. 3. Capability limits of (a) a generator neglecting machine limits (b) a DFIG, and (c) a SG

\section{B. Scenario 1: Effects of capability limits of individual DGs}

Due to the intermittent nature of wind resources and time varying load patterns, the capability chart of the microgrid also varies with time. For the purpose of analysis, the capability chart is derived for the period when the microgrid has minimum load (1.56 MW) and maximum generation (5.4 MW). Nodal voltages of the microgrid are allowed to vary within $\pm 5 \%$ and loads are modelled as constant power loads. As illustrated in Fig. 3-(b), active and reactive power capability of a DFIG based wind generator unit is mainly determined by the rotor current limit, stator current limit and the wind turbine rating [10], [11]. Similarly, power output of a SG based hydro power plant is limited by the rotor current limit, stator current limit, load angle and the hydro-turbine rating [19] as illustrated in Fig. 3-(c). However, if those machine limits are not taken into account, a simple capability chart for DG will be similar to Fig.3-(a). Fig. 4 illustrates two capability charts of

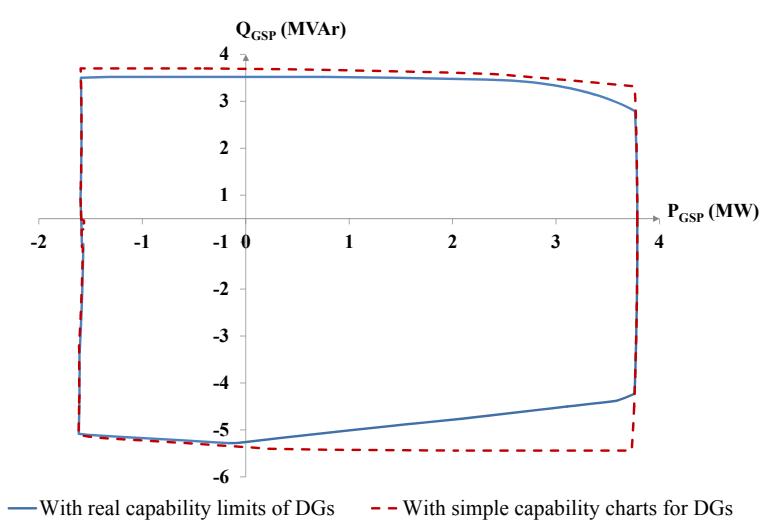

Fig. 4. Impact of simplistic and real DG capability limits on the capability chart of microgrid model-1 the microgrid, derived considering different capability limits of the DGs. Positive values indicate power export from the microgrid to the external grid, while negative values represent power import into the microgrid. It can be observed that the assumption of simplistic capability limits for DGs as in Fig. 3(a), instead of the real machine capability charts would give misleading information regarding the microgrid real and reactive power availability.

\section{Scenario 2: Effects of voltage dependency of loads}

In this scenario, effects of voltage dependent load models on the microgrid capability chart is analysed. Capability chart is derived for the same time period and network conditions as in Scenario 1 and loads are modelled as per (13) and Table I. Depending on the terminal voltage and the exponents of the models, active and reactive power absorption of the loads vary. Reactive power exponent of the load models considered in this scenario are relatively larger than the active power exponent. This affects the reactive power limits of the capability diagram compared to the active power limits as observed in Fig. 5.

$$
P=P_{\circ}\left(\frac{V}{V_{\circ}}\right)^{\alpha} \quad Q=Q_{\circ}\left(\frac{V}{V_{\circ}}\right)^{\beta}
$$

where $P_{\circ}, Q_{\circ}$ are active and reactive power at voltage $V_{\circ}$.

Table I

LOAD CHARACTERISTICS [19]

\begin{tabular}{c|c|c|c}
\hline Load class & p.f. & $\alpha$ & $\beta$ \\
\hline \hline Load 1 (industrial) & 0.85 & 0.99 & 3.5 \\
\hline Load 2 (commercial) & 0.85 & 0.18 & 6.0 \\
\hline
\end{tabular}

\section{Scenario 3: Variation of capability chart with different loading conditions}

Fig. 6 illustrates the microgrid capability for maximum load (6.3 MW) and minimum load (1.56 MW) under maximum generation $(5.4 \mathrm{MW})$ conditions. With minimum loading conditions, the microgrid has a higher capability to supply power to the upstream network and demonstrates four quadrant

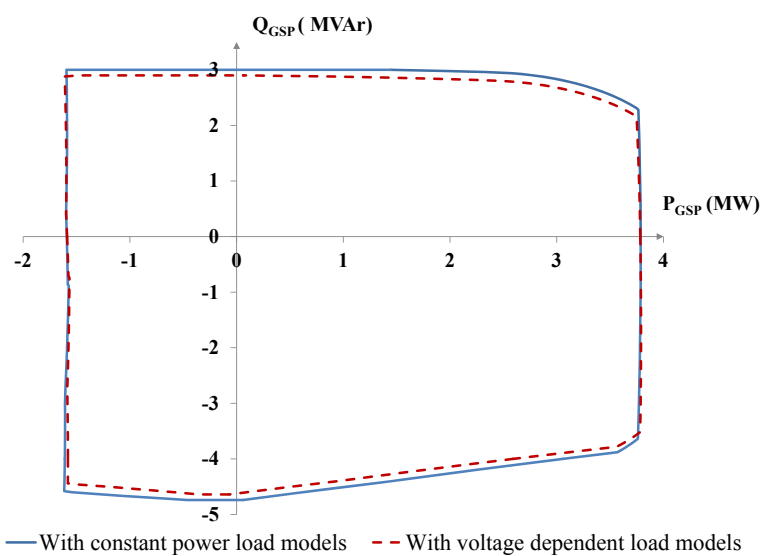

Fig. 5. Effects of voltage dependent load modelling on the capability chart of microgrid model-1 


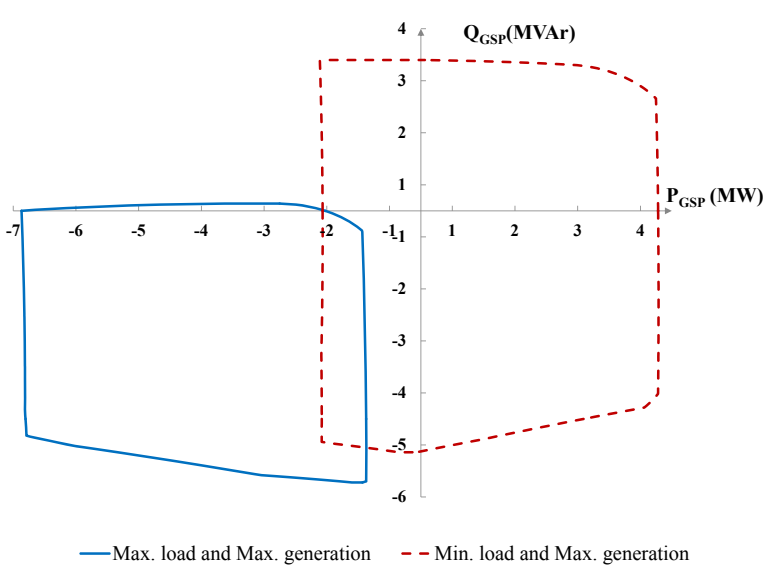

Fig. 6. Variation of capability chart for different loading conditions in microgrid model-1

operation. Whereas, with maximum loading conditions, this particular microgrid is unable to export active power to the external grid. Network losses corresponding to the capability limit during minimum loading conditions is illustrated in Fig. 7.

\section{E. Scenario 4: Effects of voltage regulation on microgrid capability limits}

In this scenario, system conditions of the microgrid are maintained similar to Scenario 2 and only the voltage regulation at the buses are varied. An increase in terminal voltage will allow DGs to output higher active power to the network. As expected, it can be observed from Fig. 8 that an increase in the range of voltage regulation is favourable for power export from the microgrid.

\section{Case Study Based on Modified IEEE 13- Bus SYSTEM}

A case study is carried on a microgrid model developed based on the modified IEEE 13-Bus system [20]. Fig. 9 illustrates the three-phase balanced microgrid model-2 comprising two DFIGs (1.5 MVA) and an SG(5 MVA). The microgrid is connected to the main grid through a $115 \mathrm{kV} / 4.16 \mathrm{kV}$, 10 MVA transformer. Generation profiles used in Section III are considered in this study and details of the load models are presented in Table II. In order to support the network voltage,

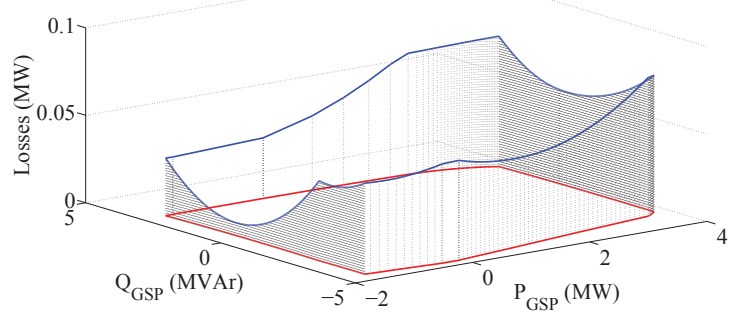

Fig. 7. Active power losses corresponding to the capability limit under minimum loading conditions of the microgrid model-1

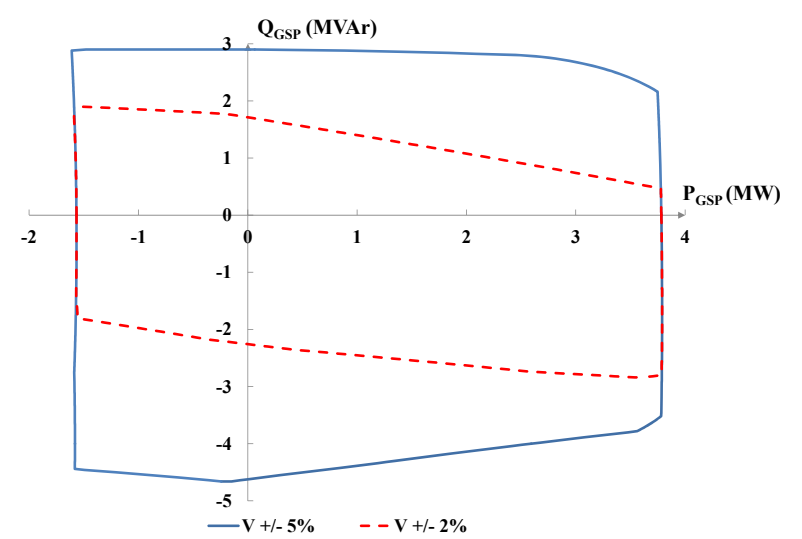

Fig. 8. Effects of voltage regulation on the capability chart of microgrid model-1

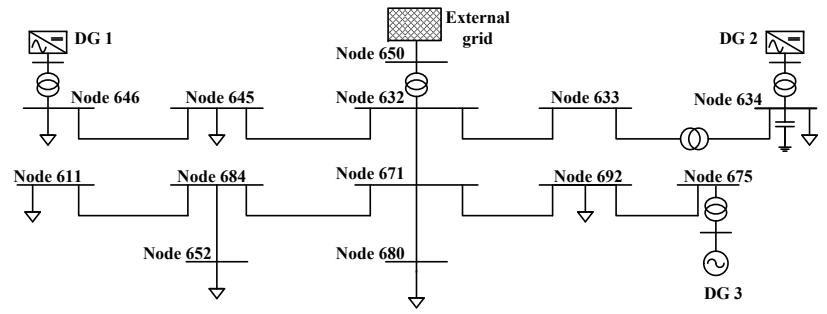

Fig. 9. Single line diagram of the microgrid model-2

a fixed capacitor bank rated at 0.5 MVAr is connected to Bus634.

A capability chart for microgrid model-2 at the GSP is obtained for maximum generation and maximum loading conditions using the optimisation model explained in Section II. The fixed capacitor bank is modelled as a constant impedance load. It can be observed from Fig. 10 that constant power load model significantly affects the capability limits at the GSP. Furthermore, unlike capability charts of individual machines, the shape of the microgrid capability chart is unique to the microgrid. Operating points on the capability limit are verified using the time domain simulation model developed in DIgSILENT PowerFactory. Fig. 11 illustrates the system losses corresponding to the capability limits of microgrid model-2.

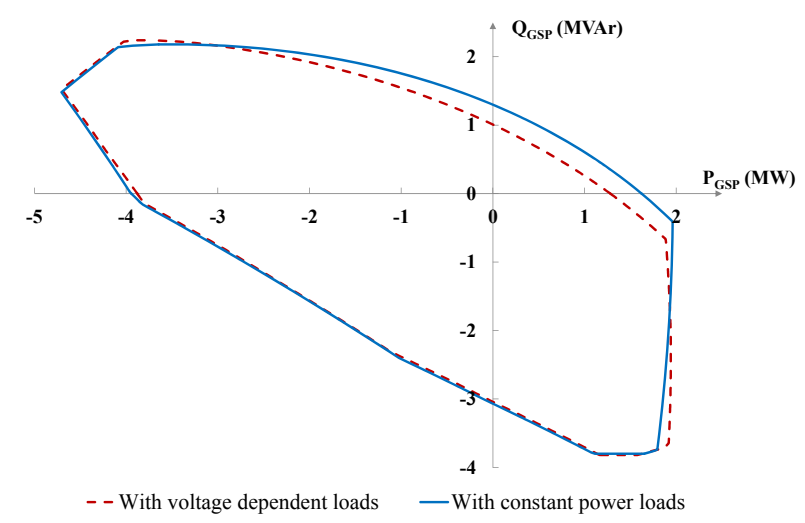

Fig. 10. Effects of voltage dependent load modelling on the capability chart of microgrid model-2 


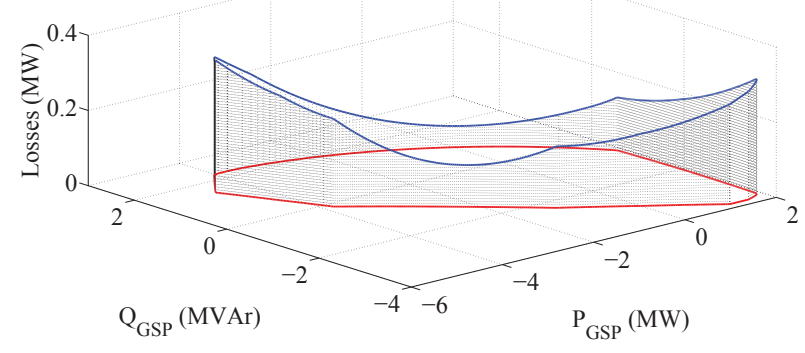

Fig. 11. System losses corresponding to the capability limits of the microgrid model-2

Table II

DETAILS OF LOADS IN MICROGRID MODEL-2 [19]

\begin{tabular}{c|c|c|c|c|c}
\hline Load-bus & Load class & $P_{\circ}(M W)$ & p.f & $\alpha$ & $\beta$ \\
\hline \hline 611 & Industrial & 0.3 & 0.85 & 0.18 & 6.0 \\
\hline 634 & Residential & 0.9 & 0.9 & 1.2 & 2.9 \\
\hline 645 & Commercial & 1.0 & 0.85 & 0.99 & 3.5 \\
\hline 646 & Residential & 0.5 & 0.9 & 1.2 & 2.9 \\
\hline 652 & Industrial motors & 0.1 & 0.88 & 0.07 & 0.5 \\
\hline 680 & Commercial & 0.5 & 0.85 & 0.99 & 3.5 \\
\hline 692 & Industrial & 1.4 & 0.85 & 0.18 & 6.0 \\
\hline \multicolumn{7}{|r|r}{}
\end{tabular}

\section{Discussion}

Storage devices have been omitted from the analysis presented in this paper. In microgrids, storage devices are utilised to mitigate the power mismatch, smooth out variability of renewable energy generations, and to reduce network losses. Furthermore, storage devices in microgrids may be used to store energy during low electricity prices and supply energy back to the microgrid when electricity market price is high. If a grid-tied microgrid is not self-sufficient in regards to other local DGs, local storage devices within the microgrid can be utilised to reduce the power exchange with the main grid. Similar to other DGs, storage devices in microgrids can sell stored excess energy to the external grid. A storage device can be modelled as a source or a load depending on its operation. However, feasibility of operating storage devices need to be carefully analysed considering technical and economic aspects prior to developing the microgrid capability chart.

Typically, capacitor banks are used to support the local voltage by injecting reactive power. In the market paradigm, reactive power available from the capacitor banks can also be exported to the external grid via the MCCs. Thus, it is proposed that the capacitor banks (switched or fixed) may be included as local DG with reactive power (only) capabilities. However, such operation requires careful analysis of economical and technical benefits prior to being incorporated in the microgrid capability chart.

\section{CONCLusions}

This paper presented a systematic approach to develop a capability chart for a grid-tied microgrid. An optimaistion model was developed and solved iteratively to obtain the capability limits for two different microgrid models. Effects of real DG capability limits, load modelling, and voltage regulation on microgrid capability was analysed. Furthermore, modelling of storage devices and capacitor banks were discussed. Depending on the requirements of the MCC or the DNSP, capability charts of grid-tied microgrids can be utilised as graphical tools which will assist to understand the microgrid behaviour, to allow optimum use of DERs and to provide coordinated support to the network through ancillary services. Capability charts can be derived online for multiple generator configurations, network configurations, and load profiles.

\section{REFERENCES}

[1] F. Farzan, S. Lahiri, M. Kleinberg, K. Gharieh, F. Farzan, and M. Jafari, "Microgrids for fun and profit: The economics of installation investments and operations," Power and Energy Magazine, IEEE, vol. 11, no. 4, pp. 52-58, July-Aug. 2013.

[2] M. Smith and D. Ton, "Key connections: The u.s. department of energy's microgrid initiative," Power and Energy Magazine, IEEE, vol. 11, no. 4, pp. 22-27, July-Aug. 2013.

[3] A. Dimeas and N. Hatziargyriou, "Operation of a multiagent system for microgrid control," IEEE Trans. Power Syst., vol. 20, no. 3, pp. 14471455, Aug. 2005.

[4] A. Tsikalakis and N. Hatziargyriou, "Centralized control for optimizing microgrids operation," IEEE Trans. Energy Convers., vol. 23, no. 1, pp. 241-248, March 2008.

[5] S. Arefifar, Y. Mohamed, and T. H. M. EL-Fouly, "Supply-adequacybased optimal construction of microgrids in smart distribution systems," IEEE Trans. Smart Grid, vol. 3, no. 3, pp. 1491-1502, Sept. 2012.

[6] A. Gabash and P. Li, "Active-reactive optimal power flow in distribution networks with embedded generation and battery storage," IEEE Trans. Power Syst., vol. 27, no. 4, pp. 2026-2035, Nov. 2012.

[7] L. Ochoa, A. Keane, and G. Harrison, "Minimizing the reactive support for distributed generation: Enhanced passive operation and smart distribution networks," IEEE Trans. Power Syst., vol. 26, no. 4, pp. 2134 2142, Nov. 2011

[8] S. Paudyal, C. Canizares, and K. Bhattacharya, "Optimal operation of distribution feeders in smart grids," IEEE Trans. Ind. Electron., vol. 58, no. 10 , pp. 4495-4503, Oct. 2011.

[9] N. E. Nilsson and J. Mercurio, "Synchronous generator capability curve testing and evaluation," IEEE Trans. Power Del., vol. 9, no. 1, pp. 414424, Jan. 1994.

[10] S. Engelhardt, I. Erlich, C. Feltes, J. Kretschmann, and F. Shewarega, "Reactive power capability of wind turbines based on doubly fed induction generators," IEEE Trans. Energy Convers., vol. 26, no. 1, pp. 364-372, March 2011.

[11] T. Lund, P. Sørensen, and J. Eek, "Reactive power capability of a wind turbine with doubly fed induction generator," Wind Energy, vol. 10, pp. 379-394, 2007.

[12] F. Delfino, G. Denegri, M. Invernizzi, R. Procopio, and G. Ronda, "A pq capability chart approach to characterize grid connected pv-units," in Integration of Wide-Scale Renewable Resources Into the Power Delivery System, CIGRE/IEEE PES Joint Symposium, July 2009.

[13] A. Losi, M. Russo, P. Verde, and D. Menniti, "Capability chart for generator-transformer units," in 8th Mediterranean Electrotechnical Conference, vol. 3, pp. 1580-1583, May 1996.

[14] J. De Silva, C. Arnold, and J. Arrillaga, "Capability chart for an hvde link," IEE Gen. Transm. Distrib., vol. 134, no. 3, pp. 181-186, May 1987.

[15] S. Abdelkader and D. Flynn, "Graphical determination of network limits for wind power integration," IET Gen. Transm. Distrib., vol. 3, no. 9, pp. 841-849, 2009.

[16] E. Chiodo, A. Losi, R. Mongelluzzo, and F. Rossi, "Capability chart for electrical power systems," IEE Gen. Transm. Distrib., vol. 139, no. 1, pp. 71-75, Jan. 1992.

[17] W. M. P. E. Gill and M. H. Wright, Practical Optimization. Academic Press, 1981.

[18] M. Cho and Y. Chen, "Fixed/switched type shunt capacitor planning of distribution systems by considering customer load patterns and simplified feeder model," IEE Gen. Transm. Distrib., vol. 144, no. 6, pp. 533-540, 1997.

[19] P. Kundur, Power System Stability and Control. McGraw-Hill, 1994.

[20] IEEE 13 Node Test Feeder. [Online]. Available: http://ewh.ieee.org/soc/ pes/dsacom/testfeeders/index.html 